\title{
Strict/Tolerant Logics Built Using Generalized Weak Kleene Logics
}

\author{
Melvin Fitting \\ The Graduate Center, City University of New York \\ Departments of Philosophy, Computer Science, Mathematics (emeritus) \\ melvin.fitting@gmail.com
}

\begin{abstract}
This paper continues my work of [12, which showed there was a broad family of many valued logics that have a strict/tolerant counterpart. Here we consider a generalization of weak Kleene three valued logic, instead of the strong version that was background for that earlier work. We explain the intuition behind that generalization, then determine a subclass of strict/tolerant structures in which a generalization of weak Kleene logic produces the same results that the strong Kleene generalization did. This paper provides much background, but is not fully selfcontained. In particular, some results from 12 are called on and are not reproved here.
\end{abstract}

Keywords: strict/tolerant logic, Kleene logics, bilattices

\section{Introduction}

There are two objectives in this paper. They are represented in the title via the phrases "strict/tolerant" and "weak Kleene". In this Introduction I discuss the background for these objectives, beginning with weak Kleene logic.

A three valued logic taking meaninglessness or nonsensicalness into account was introduced in [5]. It was independently reintroduced as a logic of gappiness in 16, where it was given a computational interpretation. Today it is known as Bochvar's logic, or more commonly as Kleene's weak three valued logic. Kleene also introduced a strong three valued logic. It too can be interpreted computationally. Roughly speaking, the strong version allows parallel computation while the weak version does not. With all respect to Bochvar, we will call the logic "weak Kleene" here. There have been several generalizations of weak Kleene introduced. For instance, see 21] and further references there. I introduced a naturally motivated family in [10], and applied this in my bilattice based work on Kripke's theory of truth, 8 and more recently 13. Here I find a new application for this family, investigating connections with strict/tolerant logics, thus extending my earlier work from 15,12 .

Strict/Tolerant logic, commonly known as ST, has generated much interest of late, 6, 3 , $25,19,2,20$. It is a three-valued logic with an unusual consequence relation. The truth tables are those of Kleene's strong three valued logic, often called $\mathrm{K}_{3}$. These tables are the same as those of Priest's logic of paradox, LP, though the intuitions are different. For the moment we use $\left\{0, \frac{1}{2}, 1\right\}$ for these truth values. The special feature of ST is that it has two designated sets. The so-called strict one is $\{1\}$, the designated set for $\mathrm{K}_{3}$, while the tolerant one is $\left\{\frac{1}{2}, 1\right\}$, the designated set for LP. A valuation validates a sequent $\Gamma \Rightarrow \Delta$ in ST provided, if it maps every formula in $\Gamma$ to a strictly designated truth value, it maps some member of $\Delta$ to a tolerantly

Australasian Journal of Logic (18:2) 2021, Article no. 3 
designated truth value. It turns out that ST validates the same sequents as classical logic, so it has been proposed as a replacement for classical logic in addressing the truth paradoxes, and in dealing with vagueness because it lacks transitivity. It has also led to much discussion on just what it means for logics to be different. If one identifies a logic with its consequence relation, ST is classical logic. But unlike classical logic, cut is not an admissible rule of ST using a local version of validity, so one really should not say they are the same. This is an important discussion, but it is not what concerns us here.

My own previous work in [12] (with the main ideas already in 15] which was written first but has not appeared yet) has to do with how general a phenomenon strict/tolerance is. It turns out that every many valued logic based on what I called a logical Morgan algebra has a strict/tolerant counterpart whose relationship with the many valued logic shares all the essential features that make the connection between ST and classical logic interesting. A Morgan algebra (my term) is like a De Morgan algebra except that distributivity is not required. A logical Morgan algebra is one with a designated set of members that constitute a prime filter. Classical propositional logic is determined by the simplest logical Morgan algebra. But also, both $\mathrm{K}_{3}$ and LP themselves are characterized by logical Morgan algebras, and so they too have their strict/tolerant counterparts. The same is true of first degree entailment, FDE, and indeed of an infinite family of many valued logics.

I subsequently realized that my conditions were stronger than needed, and the requirement that the designated set of truth values should be a prime filter could be relaxed. This will be discussed further in Section 2. No earlier results are invalidated by this, but rather the range of applicability is actually broadened.

Although it is not a fundamental issue like the one about sameness and difference for logics, the question does come up about what happens if, instead of using the strong Kleene truth tables, one uses the weak ones in creating something like ST, thus working with what are called paraconsistent weak Kleene and paracomplete weak Kleene. Both use Kleene's weak or Bochvar's truth tables, but the first has $\left\{\frac{1}{2}, 1\right\}$ as designated values. The second has $\{1\}$ designated, which was Kleene's choice. It turns out that results much like the original ones still connect this to classical logic, something that is a known result 18, 24. The question I investigate here is: to what extent does this generalize to the range of many valued logics that my work in [12] considered? The answer is, it does generalize, but to a proper subset of the original range. To be specific, things work well for a generalization of weak Kleene logic provided we have a logical Morgan algebra where the involution operation is an orthocomplement, that is, $x \vee \bar{x}=1$ and $x \wedge \bar{x}=0$. Of course all this requires one to say what counts as a natural generalization of weak Kleene logic. As mentioned above, I proposed such a thing in [10], and that will be employed here, preceded by some discussion to motivate it. I note that this generalization has been further considered in 7], with a dualized version explored in [22, 23]. We will actually make use of this dualized version, as well as of the original version. Bilattices play a central role, and we provide a summary of their basic properties, for convenience.

\section{Morgan Algebras}

Our methods work with many valued logics whose truth value space has some algebraic structure. Essentially we need De Morgan algebras, but we don't need the usual distributivity condition. The following terminology was introduced in [12, where dropping 'De' from 'De Morgan' is meant to suggest dropping distributivity.

Definition 2.1 (Morgan Algebra) A Morgan algebra is a bounded lattice with an involution. Thus it is a partial ordering with a least and a greatest member, with meets and joins for every pair, and in which there is an order reversing mapping, called an involution. We will write $\leq$ 
for the lattice ordering, 0 and 1 for the least and greatest members, $\sqcap$ and $\sqcup$ for meet and join, and overbar for the involution.

It is easy to show that in a Morgan algebra, $\overline{\bar{a}}=a, \overline{a \sqcap b}=\bar{a} \sqcup \bar{b}$, and $\overline{a \sqcup b}=\bar{a} \sqcap \bar{b}$. Also $\overline{0}=1$ and $\overline{1}=0$.

Definition 2.2 (Logical Language) We assume we have a fixed logical language, built up from propositional letters using the connectives $\wedge, \vee$, and $\neg$. We commonly use $X, Y, \ldots$ for formulas and $P, Q, \ldots$ for propositional letters. We also have sequents which we write as $\Gamma \Rightarrow \Delta$, where $\Gamma$ and $\Delta$ are finite sets of formulas.

Sequents will be treated semantically, not proof theoretically. They involve sets, not multisets, and structural rules are not considered. They are interpreted in various algebraic structures here, using valuations, where valuations are defined arbitrarily at the atomic level, and are then extended. In 12 the same notation was used for a valuation and for its extension to all formulas. Here we need to be a bit more careful since a couple of ways of extending will be used. One family of extensions makes use of the lattice structure of its truth value space - it will be denoted $v^{l}$, where the $l$ is for lattice. This is the extension used in the earlier paper. Other ways that do not use the lattice structure directly will have different notations.

Definition 2.3 (Valuation) A valuation in a Morgan algebra $L$ is a mapping $v$ from propositional letters to members of $L$. Every such valuation extends uniquely to a mapping $v^{l}$ on all formulas as follows, where $\sqcap$ and $\sqcup$ are the meet and join of the algebra.

$$
\begin{aligned}
v^{l}(P) & =v(P) \text { for } P \text { a propositional letter } \\
v^{l}(X \wedge Y) & =v^{l}(X) \sqcap v^{l}(Y) \\
v^{l}(X \vee Y) & =v^{l}(X) \sqcup v^{l}(Y) \\
v^{l}(\neg X) & =\overline{v^{l}(X)}
\end{aligned}
$$

To have a semantic characterization of a many valued logic we also need a set of designated truth values. In 12 we required that designated sets should be prime filters. After the fact it was realized that this was a much stronger condition than was needed and, in fact, the strength was never employed in our proofs. For the record, here is a brief discussion of the range of possible conditions, in order of increasing strength.

Definition 2.4 (Designated Set Conditions) Let $L=\langle L, \leq\rangle$ be a lattice. A subset $S$ of $L$ is:

1. upward closed, or just upclosed, if $x \in S$ and $x \leq y$ implies $y \in S$;

2. a filter if it is upward closed and $x, y \in S$ implies $x \sqcap y \in S$;

3. a prime filter if it is a filter and $x \sqcup y \in S$ implies $x \in S$ or $y \in S$.

If we think of the members of a lattice $L$ as a space of truth values, with the ordering relation having to do with the degree of truth, whatever that might mean, then the conditions just given have natural roles. Suppose $S$ is the set of designated truth values, those we accept as established, valid, factual, .... And suppose we map formulas into the lattice with conjunction corresponding to meet and disjunction corresponding to join. Then being upward closed says that anything truer than an accepted formula is also accepted. Being a filter adds that the accepted formulas are closed under conjunction. Being a prime filter adds that if a disjunctive formula is accepted, one of its disjuncts also is. These are natural conditions of increasing strength. In [15, 12] a condition was imposed of being a prime filter. In fact, just being upward closed would have been quite enough, and that is what is used here. 
Definition 2.5 (Logical Morgan Algebra) Let $L=\langle L, \leq\rangle$ be a Morgan algebra, and let $D$ be a non-empty, proper subset of $L$ that is upward closed. We call the pair $\langle L, D\rangle$ an upclosed logical Morgan algebra. Thinking of $L$ as a space of truth values, $D$ serves as the set of designated truth values.

We will not need the following, but it would be natural to say that an upclosed logical Morgan algebra $\langle L, D\rangle$ is filtered if $D$ is a filter, and is prime filtered if $D$ is a prime filter. In 15, 12], the terminology Logical Morgan Algebra was used. It will not be used here, but it is equivalent to being a prime filtered logical Morgan algebra

Definition 2.6 (Consequence) Let $\langle L, D\rangle$ be an upclosed logical Morgan algebra, and let $v$ be a valuation in $L . v$ validates the sequent $\Gamma \Rightarrow \Delta$ in $\langle L, D\rangle$ provided that, if $v^{l}(X) \in D$ for every $X \in \Gamma$ then $v^{l}(Y) \in D$ for some $Y \in \Delta$. A sequent $\Gamma \Rightarrow \Delta$ is valid in $\langle L, D\rangle$ if every valuation in $\langle L, D\rangle$ validates it.

\section{Bilattices}

Bilattices, like Morgan algebras, are algebraic structures whose members can be thought of as truth values, but they carry two orderings. One is commonly thought of as being on degree of truth, and the other on degree of information. They are the chief tool we use in what follows. Quite a number of different conditions have been imposed on bilattices but rather than listing the ones we need each time we talk about bilattices, we keep terminology at a minimum by stipulating that here bilattice will be short for interlaced bilattice with negation and conflation that commute. We say what all this means, and then we sketch what consequences will be needed, omitting much of the motivation and proofs. See 14, 11] for a fuller treatment (though it hardly exhausts the subject).

Definition 3.1 (Bilattice) A bilattice (in this paper) is a structure $\mathcal{B}=\left\langle\mathcal{B}, \leq_{t}, \leq_{k}\right\rangle$ with two partial orderings, $\leq_{t}$ (the truth ordering) and $\leq_{k}$ (the information ordering). These meet the following conditions.

1. Each partial ordering is that of a lattice. Meet and join with respect to $\leq_{t}$ are denoted $\wedge$ and $\vee$. Meet and join with respect to $\leq_{k}$ are denoted $\otimes$ and $\oplus$.

2. Each partial ordering is bounded. The ordering $\leq_{t}$ has a least element, $\mathbf{f}$, and a greatest, t. The ordering $\leq_{k}$ has a least element, $\perp$, and a greatest, $T$. All four are distinct.

3. The two orderings are interlaced:

(a) $x \leq_{t} y$ implies $x \otimes z \leq_{t} y \otimes z$,

(b) $x \leq_{t} y$ implies $x \oplus z \leq_{t} y \oplus z$,

(c) $x \leq_{k} y$ implies $x \wedge z \leq_{k} y \wedge z$,

(d) $x \leq_{k} y$ implies $x \vee z \leq_{k} y \vee z$.

4. There are two involutions. One is called negation and is written as $\neg x$; it reverses $\leq_{t}$ but does not change $\leq_{k}$. Thus $x \leq_{t} y$ implies $\neg y \leq_{t} \neg x$, and $x \leq_{k} y$ implies $\neg x \leq_{k} \neg y$. Since it is an involution, $\neg \neg x=x$. The other is called conflation and is written as $-x$. It has similar properties, but with the roles of $\leq_{t}$ and $\leq_{k}$ exchanged. The two operations commute, $\neg-x=-\neg x$.

It is easy to show that negation and conflation obey De Morgan laws.

$$
\begin{array}{ll}
\neg(a \wedge b)=(\neg a \vee \neg b) & -(a \otimes b)=(-a \oplus-b) \\
\neg(a \vee b)=(\neg a \wedge \neg b) & -(a \oplus b)=(-a \otimes-b)
\end{array}
$$


Likewise we have the following De Morgan-like laws.

$$
\begin{array}{ll}
-(a \wedge b)=(-a \wedge-b) & \neg(a \otimes b)=(\neg a \otimes \neg b) \\
-(a \vee b)=(-a \vee-b) & \neg(a \oplus b)=(\neg a \oplus \neg b)
\end{array}
$$

One can show the following, though we omit the proof.

$$
\begin{aligned}
\mathbf{f} \otimes \mathbf{t} & =\perp \\
\mathbf{f} \oplus \mathbf{t} & =\top \\
\perp \wedge \top & =\mathbf{f} \\
\perp \vee \top & =\mathbf{t}
\end{aligned}
$$

Since bilattice members can be thought of as generalized truth values, one expects valuations to appear. It is here that the truth ordering plays its role.

Definition 3.2 (Valuation) A valuation in a bilattice $\mathcal{B}=\left\langle\mathcal{B}, \leq_{t}, \leq_{k}\right\rangle$ is a mapping $v$ from propositional letters to members of $\mathcal{B}$. Valuations are extended to all formulas using the lattice operations of the truth ordering. This is really a special case of Definition 2.3 .

$$
\begin{aligned}
v^{l}(P) & =v(P) \text { for } P \text { a propositional letter } \\
v^{l}(X \wedge Y) & =v^{l}(X) \wedge v^{l}(Y) \\
v^{l}(X \vee Y) & =v^{l}(X) \vee v^{l}(Y) \\
v^{l}(\neg X) & =\neg v^{l}(X)
\end{aligned}
$$

Valuations are extended using the operations of the truth ordering, but the information ordering also plays a significant role here, largely because of the following monotonicity result.

Proposition 3.3 Let $v, w$ be valuations in bilattice $\mathcal{B}$. If $v(P) \leq_{k} w(P)$ for every propositional letter $P$, then $v^{l}(X) \leq_{k} w^{l}(X)$ for every formula $X$.

Proof This is an easy consequence of the last two of the interlacing conditions, and the condition that the ordering $\leq_{k}$ is preserved by the bilattice $\neg$ operation.

There is a simple way of constructing bilattices, starting with Morgan algebras.

Definition 3.4 (Bilattice Product) Let $L$ be a Morgan algebra with $\leq$ as the partial ordering and $\bar{x}$ as the involution of $x$. The bilattice product of $L$ with itself is $L \odot L=\left\langle L \times L, \leq_{t}, \leq_{k}\right\rangle$ where the structure is defined as follows.

1. $\langle a, b\rangle \leq_{t}\langle c, d\rangle$ iff $a \leq c$ and $d \leq b$

2. $\langle a, b\rangle \leq_{k}\langle c, d\rangle$ iff $a \leq c$ and $b \leq d$

3. $\neg\langle a, b\rangle=\langle b, a\rangle$

4. $-\langle a, b\rangle=\langle\bar{b}, \bar{a}\rangle$

It is always the case that a bilattice product is, in fact, a bilattice. The motivating intuition behind the construction is rather simple. Think of a pair $\langle a, b\rangle$ as encoding that there is $a$ evidence for some proposition and $b$ evidence against. Evidences for and against are allowed to be incomplete or inconsistent, where just what this means depends on details of each particular bilattice. Degree of truth goes up if evidence for goes up while evidence against goes down. (Note the reversal of the ordering of $b$ and $d$ in the definition of $\leq_{t}$.) Degree of information goes up if both the degree of evidence for and the degree of evidence against goes up. Negation 
simply switches the roles of for and against. Conflation is more complicated. If $a$ is evidence for something, think of $\bar{a}$ as its complement - that which is not evidence for that thing. Similarly for the evidence against case. Then in $-\langle a, b\rangle$ evidence for is replaced by whatever was not evidence against, and evidence against is replaced by whatever was not evidence for.

The extreme elements of a bilattice product are $\perp=\langle 0,0\rangle, \top=\langle 1,1\rangle, \mathbf{f}=\langle 0,1\rangle$, and $\mathbf{t}=\langle 1,0\rangle$, where 0 is the least member of $L$ and 1 is the greatest. Then, for instance, $\mathbf{f}$ represents no evidence for, but total evidence against. The bilattice operations are the following, where $\sqcup$ and $\sqcap$ are the lattice joins and meets.

$$
\begin{aligned}
& \langle a, b\rangle \wedge\langle c, d\rangle=\langle a \sqcap c, b \sqcup d\rangle \\
& \langle a, b\rangle \vee\langle c, d\rangle=\langle a \sqcup c, b \sqcap d\rangle \\
& \langle a, b\rangle \otimes\langle c, d\rangle=\langle a \sqcap c, b \sqcap d\rangle \\
& \langle a, b\rangle \oplus\langle c, d\rangle=\langle a \sqcup c, b \sqcup d\rangle
\end{aligned}
$$

There are representation theorems concerning bilattice products - they provide a general method of bilattice construction. If $\mathcal{B}$ is a bilattice, there is some Morgan algebra $L$ so that $\mathcal{B}$ is isomorphic to $L \odot L$. We are using a notion of bilattice with several conditions imposed. Versions of the construction apply if we work with bilattices with fewer conditions, without negation or conflation, say. What is appropriate then is a product of two different lattices, which may or may not have De Morgen involutions. In all cases the construction method is completely general. This is not simple to prove, and we do not actually need it. See [14, 11 for details and further references.

If members of bilattices are to play the role of generalized truth values, some notion of designated element is needed. In [1] prime bifilters and logical bilattices were introduced. We only need weaker versions here.

Definition 3.5 (Upbiclosed Logical Bilattices) Let $\mathcal{B}=\left\langle\mathcal{B}, \leq_{t}, \leq_{k}\right\rangle$ be a bilattice, and let $D$ be a proper, non-empty subset of $\mathcal{B}$. Recall the terminology from Definition 2.4 .

1. $D$ is upbiclosed if it is upwardly closed with respect to both orderings $\leq_{t}$ and $\leq_{k}$.

2. $\langle\mathcal{B}, D\rangle$ is an upbiclosed logical bilattice if $D$ is an upbiclosed set.

The following is of considerable use in our main construction. The proof, which is trivial, is omitted.

Proposition 3.6 Let $L$ be a Morgan algebra with $D \subseteq L$. D is upwardly closed in $L$ if and only if $D \times L$ is upbiclosed in the bilattice $L \odot L$. Consequently, $\langle L, D\rangle$ is an upclosed logical Morgan algebra if and only if $\langle L \odot L, D \times L\rangle$ is an upbiclosed logical bilattice.

Example 3.7 Figure 1 shows the simplest example of the logical bilattice construction. In Subfigure 1a is the lattice of classical logic, with ordering upward. The involution is the usual, $\overline{0}=1$ and $\overline{1}=0$. The only (proper) upwardly closed set is shown circled. (It is, in fact, a prime filter.) Applying the bilattice product construction from Definition 3.4 we get the bilattice in Subfigure 1b, where the two orderings are shown using a double Hasse diagram, which is standard with bilattices. Also, applying the product construction from Proposition 3.6 we get the two element subset shown in the oval. (It is actually what is called a prime bifilter.) This upbiclosed logical bilattice is, in fact, a standard when working with first degree entailment, FDE. In it the only members are the extreme ones, and $\mathbf{f}=\langle 0,1\rangle, \mathbf{t}=\langle 1,0\rangle, \perp=\langle 0,0\rangle$, and $\top=\langle 1,1\rangle$. 


\section{1}

(a) Classical Logic

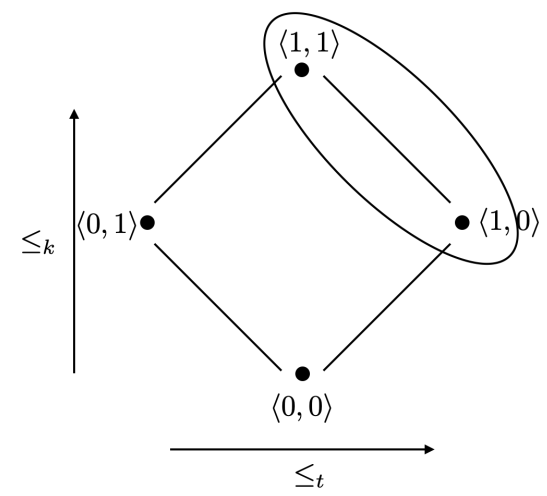

(b) The Simplest Bilattice

Figure 1: A Product Bilattice

\section{Consistent, Anticonsistent, Exact}

We return to the intuitive explanation of conflation, following Definition 3.4. In a Morgan algebra we might think of a member $a$ as representing evidence of some kind for a proposition, in which case $\bar{a}$ is simply non-evidence. Also recall, we think of a member $\langle a, b\rangle$ of a product bilattice as encoding evidence for a proposition as $a$ and evidence against as $b$. Following up on this, we might then think of a member $\langle a, b\rangle$ of a product bilattice as "consistent" if $a$ and $b$ don't conflict - the evidence for, $a$, can encode no evidence against, but non-evidence against is allowed. That is, we should have $a \leq \bar{b}$. In fact, it is easy to see that $a \leq \bar{b}$ and $b \leq \bar{a}$ are equivalent in a Morgan algebra, and both are equivalent to $\langle a, b\rangle \leq_{k}-\langle a, b\rangle$ in the corresponding product bilattice. This, and other similar considerations, lead us to the following.

Definition 4.1 (Consistent, Anticonsistent, Exact) In a bilattice $\mathcal{B}$, a member $x$ is:

1. consistent if $x \leq_{k}-x$,

2. anticonsistent if $-x \leq_{k} x$,

3. exact if $x=-x$.

The categories consistent and exact come from [8], while anticonsistent waited until [14, 11] and [13]. It is not the case that every member of a bilattice must fall into one of these three categories; the categories are, in a sense, the well-behaved parts. Here are some important and useful properties of consistency, anticonsistency, and exactness. They are generally easy to establish, and proofs can be found in [12. The notion of sharpening originated in 20], specifically for ST, but has been extended and moved to bilattices in our work. For two valuations $u, v$ mapping to the anticonsistent part of a bilattice, $u$ sharpens $v$ if, for every propositional letter $P, u(P)$ is exact and $u(P) \leq_{k} v(P)$.

Proposition 4.2 In any bilattice $\mathcal{B}$ we have the following.

1. The sets of exact values, consistent values, and anticonsistent values in $\mathcal{B}$ are closed under $\wedge, \vee$, and $\neg$.

2. The sets of exact values, consistent values, and anticonsistent values in $\mathcal{B}$ each contain $\mathbf{f}$ and $\mathbf{t}$. 
3. In $\mathcal{B}, \perp$ is consistent and $\top$ is anticonsistent.

4. Every consistent value in $\mathcal{B}$ is below some exact value, and every anticonsistent value is above some exact value, in the $\leq_{k}$ ordering.

5. For exact $a$ and $b$ in $\mathcal{B}$, if $a \leq_{k} b$ then $a=b$.

6. If valuation $v$ maps every propositional letter to a consistent truth value in $\mathcal{B}$, its extension to all logical formulas, $v^{l}$, maps every formula to a consistent truth value. Similarly for the exact truth values, and for the anticonsistent truth values.

7. Every valuation $v$ mapping propositional letters to the anticonsistent part of $\mathcal{B}$ has a sharpening.

8. If $u$ sharpens $v$ in $\mathcal{B}$ then $u$ and $v$ agree on exact values. That is, if $u$ sharpens $v$ and $u(P)$ and $v(P)$ are both exact, then $u(P)=v(P)$.

Returning to the simplest bilattice example in Figure $1 \mathrm{~b}$, it is actually a remarkable structure. Using the $\leq_{t}$ order, it provides the semantics for first degree entailment. The consistent part is $\{\mathbf{f}, \perp, \mathbf{t}\}$ which is closed under the truth based operations. Together with the displayed prime bifilter restricted to this subset, we have the structure for Kleene's strong three valued logic, $\mathrm{K}_{3}$. The anticonsistent part is $\{\mathbf{f}, \top, \mathbf{t}\}$, also closed under the truth operations. Using the prime bifilter restricted to this subset, we have the structure for Priest's logic of paradox, LP. Finally, the exact part is $\{\mathbf{f}, \mathbf{t}\}$, also closed under the truth operations. Using the prime bifilter, restricted to the exact subset, as providing the designated set, the exact part gives us an isomorphic copy of the classical structure from Figure $1 \mathrm{a}$

All this suggests that consistent parts of bilattices make up a natural family of generalizations for Kleene's strong three valued logic. ([9] should also be consulted on this point.) Similarly for anticonsistent parts of bilattices as generalizations of the logic of paradox. For bilattices that are complete, in the sense that they are closed under infinite meets and joins, Kripke style bilattice generalizations of fixpoint theories of truth work out quite nicely, see [8, 13].

\section{How Weak Kleene Logic Generalizes}

Generalizations of strong Kleene logic arise naturally from bilattices, as the subsets of consistent values in them. In 10] I proposed a plausible way of also using bilattices to create generalizations of weak Kleene logic, and I will repeat it here, beginning with some background intuition. See

7. for further work on this.

Suppose we have a set of agents $\mathcal{A}$, each of whom can vote for the truth of some proposition, and quite independently vote against its truth. They are allowed to vote both ways, that is, to be contradictory. They are also allowed to abstain from voting at all. The acceptance status of a proposition can be represented as a pair, $\langle F, A\rangle$, where $F$ is the set of agents voting for the proposition, and $A$ is the set voting against. That is, it is a member of the product bilattice $\mathcal{P}(\mathcal{A}) \odot \mathcal{P}(\mathcal{A})$, where $\mathcal{P}$ is the powerset operation, and we think of $\mathcal{P}(\mathcal{A})$ as a Morgan (actually, De Morgan) algebra partially ordered by subset, with relative complement as involution. But now suppose we are only interested in those agents who are willing to express their opinions fully. So, for instance, if an agent does not vote at all on proposition $X$, we discount the agent's opinion on $X \vee Y$. In more detail, let us say the acceptance status assigned to $X$ is $\langle A, B\rangle$. Then if an agent does not turn up in $A \cup B$, we do not record anything for that agent on $X \vee Y$, even if the agent expressed an opinion on $Y$. A simple way to represent this is to apply standard bilattice machinery to compute a value for $X \vee Y$, and then 'cut it down' by applying the consensus operator, $\otimes$, to restrict this value to $\langle A \cup B, A \cup B\rangle$. But, $\langle A \cup B, A \cup B\rangle$ is easily expressed using bilattice machinery, as $\langle A, B\rangle \oplus \neg\langle A, B\rangle$. All this leads us to Definition 5.1 . where $\wedge^{c}$ and $\vee^{c}$ can be thought of as the usual bilattice operations cut down to those agents who actually appear either for or against in both inputs to the operation. 
Kleene's original motivation was in terms of computational processes that might or might not terminate. The motivation above could be rephrased in these terms too, but probably the idea is sufficiently clear given what has been said, and we avoid the additional complexities.

Definition 5.1 (Cut Down Operations) Let $\mathcal{B}$ be a bilattice. For $x, y \in \mathcal{B}$ :

$$
\begin{aligned}
\|x\| & =x \oplus \neg x \\
x \wedge^{c} y & =(x \wedge y) \otimes\|x\| \otimes\|y\| \\
x \vee^{c} y & =(x \vee y) \otimes\|x\| \otimes\|y\| .
\end{aligned}
$$

Dualized versions of cut down operations were studied in 22, 23, under the name track down operations. These will play a role here as well. (Notation here is not what was used in the cited papers.)

Definition 5.2 (Track Down Operations) Let $\mathcal{B}$ be a bilattice. For $x, y \in \mathcal{B}$ :

$$
\begin{aligned}
|x| & =x \otimes \neg x \\
x \wedge^{t} y & =(x \wedge y) \oplus|x| \oplus|y| \\
x \vee^{t} y & =(x \vee y) \oplus|x| \oplus|y| .
\end{aligned}
$$

For both the cut down and the track down cases a negation operation could be defined: $\neg \neg^{c} x=\neg x \otimes\|x\|$ and $\neg{ }^{t} x=\neg x \oplus|x|$, but it is easy to show that both just evaluate to $\neg x$, so this is what we will use. The following gives us appropriate closure properties.

Proposition 5.3 In any bilattice, the consistent members are closed under the cut down operations and the anticonsistent members are closed under the track down operations.

Proof Suppose both $x$ and $y$ are consistent. Then $x \leq_{k}-x$ and $y \leq_{k}-y$, so $x \wedge y \leq_{k}-x \wedge-y=$ $-(x \wedge y)$, so $x \wedge y$ is consistent. And then

$$
\begin{aligned}
x \wedge^{c} y & =(x \wedge y) \otimes\|x\| \otimes\|y\| \\
& \leq_{k}(x \wedge y) \\
& \leq_{k}-(x \wedge y) \\
& \leq_{k}-(x \wedge y) \oplus-\|x\| \oplus-\|y\| \\
& =-[(x \wedge y) \otimes\|x\| \otimes\|y\|] \\
& =-\left(x \wedge^{c} y\right)
\end{aligned}
$$

so $x \wedge^{c} y$ is consistent. All the other cases are similar.

Proposition 5.4 In any bilattice:

$$
\begin{aligned}
\|x\| & =\|\neg x\|=\neg\|x\| \\
|x| & =|\neg x|=\neg|x| \\
\|-x\| & =-|x| \\
|-x| & =-\|x\| \\
\|\mathbf{t}\|=\|\mathbf{f}\|=\|\top\| & =\top \\
\|\perp\| & =\perp \\
|\mathbf{t}|=|\mathbf{f}|=|\perp| & =\perp \\
|\top| & =\top .
\end{aligned}
$$

Australasian Journal of Logic (18:2) 2021, Article no. 3 
Proof We show three items; the rest are similar.

$$
\begin{aligned}
\|\neg x\| & =\neg x \oplus \neg \neg x=\neg(x \oplus \neg x)=\neg\|x\| \\
& =\neg x \oplus \neg \neg x=\neg x \oplus x=\|x\| \\
-\|x\| & =-(x \oplus \neg x)=-x \otimes-\neg x=-x \otimes \neg-x=|-x| \\
\|\mathbf{t}\| & =\mathbf{t} \oplus \neg \mathbf{t}=\mathbf{t} \oplus \mathbf{f}=\top
\end{aligned}
$$

Proposition 5.5 In any bilattice the cut down and the track down operations obey the De Morgan laws:

$$
\begin{aligned}
& \neg\left(x \wedge^{c} y\right)=\neg x \vee^{c} \neg y \\
& \neg\left(x \vee^{c} y\right)=\neg x \wedge^{c} \neg y \\
& \neg\left(x \wedge^{t} y\right)=\neg x \vee^{t} \neg y \\
& \neg\left(x \vee^{t} y\right)=\neg x \wedge^{t} \neg y
\end{aligned}
$$

Proof We show one case.

$$
\begin{aligned}
\neg x \wedge^{c} \neg y & =(\neg x \wedge \neg y) \otimes\|\neg x\| \otimes\|\neg y\| \\
& =\neg(x \vee y) \otimes \neg\|x\| \otimes \neg\|y\| \\
& =\neg[(x \vee y) \otimes\|x\| \otimes\|y\|] \\
& =\neg\left(x \vee^{c} y\right)
\end{aligned}
$$

In Definition 3.2 a valuation into a bilattice $\mathcal{B}$ was defined to be a mapping from propositional letters to members of the bilattice. Valuations were extended to all formulas using the lattice operations associated with $\leq_{t}$. Now we add two more ways of extending.

Definition 5.6 (Valuations) Let $v$ be a valuation in bilattice $\mathcal{B}$. A cut down valuation extension $v^{c}$ and a track down extension $v^{t}$ are defined as follows.

$$
\begin{aligned}
v^{c}(P) & =v(P) \text { atomic case } & v^{t}(P) & =v(P) \text { atomic case } \\
v^{c}(X \wedge Y) & =v^{c}(X) \wedge^{c} v^{c}(Y) & v^{t}(X \wedge Y) & =v^{t}(X) \wedge^{t} v^{t}(Y) \\
v^{c}(X \vee Y) & =v^{c}(X) \vee^{c} v^{c}(Y) & v^{t}(X \vee Y) & =v^{t}(X) \vee^{t} v^{t}(Y) \\
v^{c}(\neg X) & =\neg v^{c}(X) & v^{t}(\neg X) & =\neg v^{t}(X)
\end{aligned}
$$

Proposition 5.7 If $v$, $u$ are valuations in a bilattice and $v(P)=-u(P)$ for all propositional letters $P$, then $v^{c}(X)=-u^{t}(X)$ for all formulas $X$.

Proof The proof is by induction on formula complexity; we give one case as representative. Proposition 5.4 is used here.

Suppose $v^{c}(X)=-u^{t}(X)$ and $v^{c}(Y)=-u^{t}(Y)$. We show $v^{c}(X \wedge Y)=-u^{t}(X \wedge Y)$.

$$
\begin{aligned}
v^{c}(X \wedge Y) & =v^{c}(X) \wedge^{c} v^{c}(Y) \\
& =\left(v^{c}(X) \wedge v^{c}(Y)\right) \otimes\left\|v^{c}(X)\right\| \otimes\left\|v^{c}(Y)\right\| \\
& =\left(-u^{t}(X) \wedge-u^{t}(Y)\right) \otimes\left\|-u^{t}(X)\right\| \otimes\left\|-u^{t}(Y)\right\| \\
& =-\left(u^{t}(X) \wedge u^{t}(Y)\right) \otimes-\left|u^{t}(X)\right| \otimes-\left|u^{t}(Y)\right| \\
& =-\left[\left(u^{t}(X) \wedge u^{t}(Y)\right) \oplus\left|u^{t}(X)\right| \oplus\left|u^{t}(Y)\right|\right] \\
& =-u^{t}(X \wedge Y)
\end{aligned}
$$


We have proposed that the cut down operations, on the consistent parts of bilattices, be seen as natural generalizations of Kleene's weak three valued logic. The Proposition above says that the track down operations on the anticonsistent parts could also serve, since cut down and track down have isomorphic behavior by the Proposition above.

Proposition 5.8 In any bilattice, $v^{c}(X) \leq_{k} v^{l}(X) \leq_{k} v^{t}(X)$.

Proof Similar to those above.

\section{Ways Weak Kleene Logic Generalizes}

At the beginning of Section 5 we discussed motivation for the cut down operations, in terms of agents. As a specific example along the lines of that motivation, suppose we have just a single agent. Then the powerset space has two members, the corresponding product bilattice has four members, and the consistent part three. On the consistent part the bilattice operations $\wedge, \vee$, and $\neg$ are those of Kleene's strong three valued logic, and the cut down versions are those of Kleene's weak three valued logic. The point is, a wide range of examples are easy to come by, and so we have an infinite family of generalizations of the strong and weak Kleene logics. This is already enough for important applications. For instance, Kripke's fixpoint theory of truth, 17] was originally presented using both strong and weak Kleene logics, and all that fixpoint theory extends directly to our generalizations, provided we add an assumption of completeness, that is, existence of infinite meets and joins, 8, 13.

Nonetheless, for connections with strict/tolerant logics something more is needed. Proposition 5.3 gives us closure of the consistent members of a bilattice under the cut down operationsour proposed weak Kleene generalization. But weak Kleene itself, the three valued logic, has an additional property. The subset of classical truth values, $\mathbf{t}$ and $\mathbf{f}$, behaves classically under the weak Kleene operations. Our generalization of the classical values is what we called exact in Definition 4.1. but Example 6.1 shows we can run into problems. The exact values can fail to be closed under our generalized weak Kleene connectives.

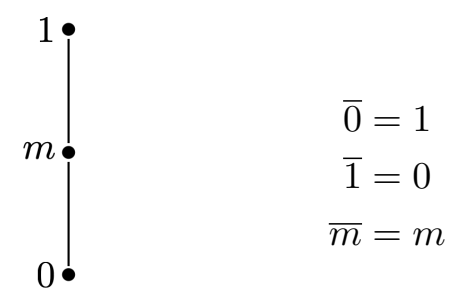

Figure 2: A Three Element Morgan Algebra

Example 6.1 In Figure 2 a Morgan algebra is shown - it is the structure underlying both $\mathrm{K}_{3}$ and LP. Just for this example, we refer to it as $M$. The bilattice $M \odot M$ contains 9 members. It is easy to check that $\langle 0,0\rangle,\langle 0, m\rangle,\langle m, 0\rangle$ are consistent but not exact, $\langle 1,1\rangle,\langle m, 1\rangle,\langle 1, m\rangle$ are anticonsistent but not exact, and $\langle 0,1\rangle,\langle 1,0\rangle,\langle m, m\rangle$ are exact.

Two exact members are $\langle 0,1\rangle$ and $\langle m, m\rangle$. A simple calculation shows that $\langle 0,1\rangle \wedge^{c}\langle m, m\rangle=$ $\langle 0, m\rangle$, which is consistent but not exact. 
Cut down operations are naturally thought of as operations on the consistent part of a bilattice. Their behavior on the anticonsistent part can be ignored. Dually for the track down operations, which really live on the anticonsistent part. But, behavior on the exact part is important. Weak and paraconsistent weak operations should agree with the underlying lattice operations on exact values. As Example 6.1 shows, this does not always happen, and what follows provides an important criterion for when it does.

Definition 6.2 A Morgan algebra $L$ is orthocomplemented if, for all $x \in L, x \wedge \bar{x}=0$ and $x \vee \bar{x}=1$.

Note: an orthocomplemented Morgan algebra that is distributive (that is, an orthocomplemented De Morgan algebra) is a Boolean algebra. Also, it is easy to show that either of the two conditions implies the other in a Morgan algebra.

Proposition 6.3 Let $L$ be a Morgan algebra, and let $\mathcal{B}=L \odot L$ be the product bilattice. If the exact members of $\mathcal{B}$ are closed under the cut down operations, then $L$ is orthocomplemented. Similarly for the track down operations.

Proof Suppose the exact members of $\mathcal{B}$ are closed under $\vee^{c}$. Let $a \in L$ be arbitrary. Then $A=\langle a, \bar{a}\rangle$ is exact. Also $\mathbf{t}=\langle 1,0\rangle$ is exact, where 0,1 are the extreme members of $L$. By our assumption, $A \vee^{c} \mathbf{t}$ is exact. Now we have the following computation.

$$
\begin{aligned}
A \vee^{c} \mathbf{t} & =(A \vee \mathbf{t}) \otimes\|A\| \otimes\|\mathbf{t}\| \\
& =\mathbf{t} \otimes\|A\| \otimes \top(\text { Proposition 5.4 } \\
& =\mathbf{t} \otimes\|A\| \\
& =\mathbf{t} \otimes(A \oplus \neg A) \\
& =\mathbf{t} \otimes(\langle a, \bar{a}\rangle \oplus \neg\langle a, \bar{a}\rangle) \\
& =\mathbf{t} \otimes(\langle a, \bar{a}\rangle \oplus\langle\bar{a}, a\rangle) \\
& =\langle 1,0\rangle \otimes\langle a \vee \bar{a}, a \vee \bar{a}\rangle \\
& =\langle a \vee \bar{a}, 0\rangle .
\end{aligned}
$$

Then by exactness $A \vee^{c} \mathbf{t}=-\left\langle A \vee^{c} \mathbf{t}\right\rangle$, so also

$$
\begin{aligned}
A \vee^{c} \mathbf{t} & =-\langle a \vee \bar{a}, 0\rangle \\
& =\langle\overline{0}, \overline{a \vee \bar{a}}\rangle \\
& =\langle 1, \bar{a} \wedge a\rangle .
\end{aligned}
$$

It follows that $a \vee \bar{a}=1$, and $a \wedge \bar{a}=0$. A similar argument can be given for the track down operations.

And in the converse direction.

Proposition 6.4 Let $L$ be a Morgan algebra that is orthocomplemented, and let $\mathcal{B}=L \odot L$. In $\mathcal{B}$ the exact values are closed under the cut down operations and the track down operations. Further, the cut down and the track down operations both agree with the bilattice $\wedge$ and $\vee$ operations on the exact values.

Proof Assume $L$ is orthocomplemented. Suppose $A$ is exact in $L \odot L$. Then $A=\langle a, \bar{a}\rangle$ for some $a \in L$. Hence $\|A\|=A \oplus \neg A=\langle a, \bar{a}\rangle \oplus\langle\bar{a}, a\rangle=\langle a \vee \bar{a}, a \vee \bar{a}\rangle=\langle 1,1\rangle=\top$. Likewise 
$|A|=\langle a, \bar{a}\rangle \otimes\langle\bar{a}, a\rangle=\langle a \wedge \bar{a}, a \wedge \bar{a}\rangle=\langle 0,0\rangle=\perp$, Then we have the following, for $A$ and $B$ exact.

$$
\begin{aligned}
A \vee^{c} B & =(A \vee B) \otimes\|A\| \otimes\|B\| \\
& =(A \vee B) \otimes \top \otimes \top \\
& =A \vee B .
\end{aligned}
$$

Thus $\vee^{c}$ and $\vee$ agree on exact values. Closure under these operations follows by Proposition 4.2 part 1. Similarly for the other cases.

The consequence we draw from the work above is simple. For some purposes, including in what follows, Morgan algebras may be too broad a class of many valued logics, and orthocomplementation is a natural additional requirement. Here is a summary of the basics so far.

Proposition 6.5 Let $v$ be a valuation in bilattice $\mathcal{B}$.

1. If $v$ maps every propositional letter to a consistent truth value, then $v^{c}$ maps every logical formula to a consistent truth value.

2. if $v$ maps every propositional letter to an anticonsistent truth value, then $v^{t}$ maps every logical formula to an anticonsistent truth value.

3. Assume $\mathcal{B}=L \odot L$, where $L$ is orthocomplemented. If $v$ maps every propositional letter to an exact truth value then both $v^{c}$ and $v^{t}$ map every formula to an exact truth value, and agree with each other and with $v^{l}$.

Proof Parts 1 and 2 are by induction on formula degree, using Proposition 5.3. Part 3 is by Proposition 6.4.

\section{Central Results}

In 12 it was shown how one could construct a strict/tolerant structure, starting with what was there called a logical Morgan algebra, so that the two would determine the same consequence relation but differ at the metaconsequence level. The same construction is used here, but with the acknowledgment that the earlier assumptions were stronger than what was actually used. We repeat the steps from the earlier paper, somewhat informally, for reference. Then we show that we still have the same results even if the strict/tolerant model uses a generalized weak Kleene logic, provided we start with a Morgan algebra that is orthocomplemented. Some examples will be found in Section 8

There is one technical point that needs to be discussed before presenting the details. The cut down operations are natural on the consistent part of a bilattice, and this is where we might expect weak Kleene generalizations to live. But the construction in 12 takes place in the anticonsistent part. There are reasons for this, but they are essentially technical. The difference is not fundamental, since the consistent and anticonsistent parts are isomorphic under the mapping $x \mapsto-x$, a mapping that preserves conjunctions, disjunctions, and negations (in particular, note Proposition 5.7). We continue to work with the anticonsistent part of a bilattice here, but we use track down operations instead of cut down ones as our generalization of the weak Kleene logic.

The construction goes as follows. Start with an upclosed logical Morgan algebra, $\langle L, D\rangle$. Using it, create the bilattice product, $L \odot L$. Then $D \times L$ will be an upbiclosed set on $L \odot L$, so we have a upbiclosed logical bilattice $\langle L \odot L, D \times L\rangle$, Definition 3.5 .

Extract a strict/tolerant logic from the upbiclosed logical bilattice as follows. The domain of truth values, call it $\mathcal{A}(L)$, is the anticonsistent part of $L \odot L$. Unlike in [12], the $\leq_{t}$ bilattice ordering is not used directly to supply meanings for conjunction and disjunction. Instead the 
track down operations $\wedge^{t}$ and $\vee^{t}$ of $L \odot L$, restricted to the anticonsistent values $\mathcal{A}(L)$, are used. Negation is interpreted by the negation operation $\neg$ of $L \odot L$ restricted to $\mathcal{A}(L)$. The tolerantly designated members, call this set $T$, are the members of $\mathcal{A}(L)$ that are in $D \times L$, that is, they are the anticonsistent members of the upbiclosed $D \times L$. The strictly designated members, denoted $S$, are the exact members of $D \times L$. Thus we have a strict/tolerant structure $\langle\mathcal{A}(L), S, T\rangle$ in which the connectives are interpreted using valuations $v^{t}$ from Definition 5.6. restricted to anticonsistent members. Note that $\mathcal{A}(L)$ is closed under the track down operations, by Proposition 5.3 .

Also extract a many valued structure from the bilattice. The domain, call it $\mathcal{E}(L)$, is the exact part of $L \odot L$. By Proposition 6.4 a lattice based interpretation for the logical connectives and a generalized weak Kleene interpretation agree on $\mathcal{E}(L)$. The designated set of values is the set $D \times L$ restricted to $\mathcal{E}(L)$. Thus the designated set here is just $S$, which is the strictly designated set for the strict/tolerant structure as well. We now have a many valued logic $\langle\mathcal{E}(L), S\rangle$. It is important to recall that, by Proposition 3 of [12], this structure is isomorphic to the upbiclosed logical Morgan algebra $\langle L, D\rangle$ that we began with. In the earlier paper I simply replaced $\langle\mathcal{E}(L), S\rangle$ with $\langle L, D\rangle$, which simplified descriptions somewhat. Here I keep both, but rely on the isomorphism.

Lemma 7.1 Every sequent not valid in the many valued structure $\langle\mathcal{E}(L), S\rangle$ is not valid in the weak Kleene strict/tolerant structure $\langle\mathcal{A}(L), S, T\rangle$.

Proof Assume the sequent $\Gamma \Rightarrow \Delta$ is not valid in the many valued structure $\langle\mathcal{E}(L), S\rangle$. We show it is not valid in $\langle\mathcal{A}(L), S, T\rangle$ in the strict/tolerant sense, using the track down operations.

Let $v$ be a valuation in $\langle\mathcal{E}(L), S\rangle$ that invalidates $\Gamma \Rightarrow \Delta$. That is, $v^{l}(X) \in S$ for all $X \in \Gamma$ but $v^{l}(Y) \notin S$ for every $Y \in \Delta$. Since $v$ is a mapping to $\mathcal{E}(L)$, it maps propositional letters to exact values. Then by Proposition 6.5 part $3, v^{l}$ and $v^{t}$ agree, and map all formulas to exact values. Then $v^{t}$ maps all members of $\Gamma$ to $S$, and no member of $\Delta$ to $S$. Since values of $v^{t}$ are exact, and $S$ is the exact subset of $T, v^{t}$ can map no member of $\Delta$ to $T$ either. This shows that $v$ invalidates $\Gamma \Rightarrow \Delta$ in $\langle\mathcal{A}(L), S, T\rangle$ as well.

Lemma 7.2 Every sequent not valid in the weak Kleene strict/tolerant structure $\langle\mathcal{A}(L), S, T\rangle$ is also not valid in the many valued structure $\langle\mathcal{E}(L), S\rangle$, and hence not valid in $\langle L, D\rangle$.

Proof Assume the sequent $\Gamma \Rightarrow \Delta$ is not valid in $\langle\mathcal{A}(L), S, T\rangle$ in the strict/tolerant sense. Then there is a valuation $v$ in $\mathcal{A}(L)$ that does not validate the sequent. That is, $v^{t}(X) \in S$ for every $X \in \Gamma$, but for every $Y \in \Delta, v^{t}(Y) \notin T$, and so for every $Y \in \Delta, v^{t}(Y)$ is anticonsistent but not in the upbiclosed $D \times L$. By Proposition 4.2 part 7, $v$ has a sharpening; choose one and call it $u$. Then $u$ is a valuation in $\langle\mathcal{E}(L), S\rangle$. We show that $u$ does not validate $\Gamma \Rightarrow \Delta$ in $\langle\mathcal{E}(L), S\rangle$, and so the sequent is not valid in $\langle L, D\rangle$.

Since the sharpening $u$ maps every propositional letter to an exact value, $u^{t}$ maps every every logical formula to an exact value, by Proposition 6.5 part 3. Also $v^{t}$ maps members of $\Gamma$ to $S$ and hence to exact values. Then by Proposition 4.2 part $8, u^{t}$ and $v^{t}$ must agree on members of $\Gamma$, and so $u^{t}$ maps every member of $\Gamma$ to $S$.

For each formula $Y \in \Delta$ we have that $v^{t}(Y)$ is anticonsistent but not in $D \times L$. If we had that $u^{t}(Y) \in S$ then $u^{t}(Y)$ would be in the upbiclosed $D \times L$. Being in this upbiclosed set we would also have $v^{t}(Y)$ in the upbiclosed because $u^{t}(Y) \leq_{k} v^{t}(Y)$ and upbiclosed sets are upward closed in both bilattice orderings. But $v^{t}(Y)$ is not in $D \times L$. Hence $u^{t}(Y) \notin S$ for every $Y \in \Delta$. It follows that $u$ does not validate $\Gamma \Rightarrow \Delta$ in $\langle\mathcal{E}(L), S\rangle$.

These Lemmas establish the following. 
Proposition 7.3 The strict/tolerant structure $\langle\mathcal{A}(L), S, T\rangle$, using the track down operations as our weak Kleene generalization, and the many valued structure $\langle\mathcal{E}(L), S\rangle$ validate the same sequents.

Now the other half of the picture. The proof is actually identical to the corresponding one in 12 .

Proposition 7.4 The metaconsequence scheme

$$
\frac{\Gamma, A \Rightarrow \Delta \quad \Gamma \Rightarrow \Delta, A}{\Gamma \Rightarrow \Delta}
$$

is locally valid in $\langle\mathcal{E}(L), S\rangle$, but an instance is not locally valid in $\langle\mathcal{A}(L), S, T\rangle$.

Proof First we show local validity in $\langle\mathcal{E}(L), S\rangle$, by showing the contrapositive. Suppose $v$ is a valuation in $\langle\mathcal{E}(L), S\rangle$ that does not validate the sequent $\Gamma \Rightarrow \Delta$ there. Then for every $X \in \Gamma$, $v^{l}(X) \in S$, and for every $Y \in \Delta, v^{l}(Y) \notin S$. Either $v^{l}(A) \in S$ or $v^{l}(A) \notin S$. If the first, then $v^{l}(X) \in S$ for every $X$ in $\Gamma, A$, so $v$ does not validate $\Gamma, A \Rightarrow \Delta$ in $\langle\mathcal{E}(L), S\rangle$. If the second, then $v^{l}(Y) \notin S$ for every $Y$ in $\Delta, A$, so $v$ does not validate $\Gamma \Rightarrow \Delta, A$ in $\langle\mathcal{E}(L), S\rangle$. Either way, $v$ does not validate one of the premises of the metaconsequence.

Second we show local non-validity in $\langle\mathcal{A}(L), S, T\rangle$. Pick any sequent $\Gamma \Rightarrow \Delta$ that is not valid in the strict/tolerant structure $\langle\mathcal{A}(L), S, T\rangle$ using the track down operations. $(\emptyset \Rightarrow \emptyset$, for instance) and let $v$ be a valuation that does not validate it. That is, $v^{t}$ maps to anticonsistent members of $L \odot L, v^{t}(X) \in S$ for every $X \in \Gamma$, and $v^{t}(Y) \notin T$ for every $Y \in \Gamma$.

Let $P$ be a propositional letter that does not occur in $\Gamma$ or in $\Delta$ and redefine $v$ so that $v(P)=\top$, where $\top$ is the largest member of $L \odot L$ in the $\leq_{k}$ ordering. This can be done without changing the behavior of $v$ on $\Gamma$ or $\Delta$. We have that $T$ is in the upbiclosed $D \times L$ because we have upward closure in both orderings. Also $T$ is anticonsistent, but not exact. Then $v$ validates $\Gamma, P \Rightarrow \Delta$ in $\langle\mathcal{A}(L), S, T\rangle$ because $v^{t}(P) \notin S$, since $v^{t}(P)$ is not exact. Also $v$ validates $\Gamma \Rightarrow \Delta, P$ in $\langle\mathcal{A}(L), S, T\rangle$ because $v^{t}(P)=\top \in T$, since $T$ is anticonsistent. So $v$ is a counterexample to the local validity of the metaconsequence scheme, taking $A$ to be the propositional letter $P$.

\section{Examples}

It may help grasp the basics of what we have been discussing if we look at some examples.

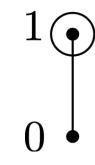

(a) Classical Logic

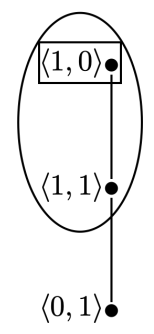

(b) Weak Kleene Logic

Figure 3: The Original Example 
Example 8.1 (Classical Logic) It is well known that weak Kleene logic has no validities, but this is when it is considered as a many valued logic with truth as the only designated value. Paraconsistent weak Kleene logic uses the same truth tables, but allows two designated truth values, and it does have validities. The strict/tolerant construction given here produces, when applied to the classical structure, a structure using precisely these two designated sets. Here is a quick sketch.

Figure 1. from earlier in this paper, shows the classical logic structure and the bilattice it generates, the usual setting for first degree entailment. The standard designated set is shown as a circled value in Subfigure 1a and it generates the upbiclosed set shown in Subfigure 1b. The anticonsistent subset for the bilattice is $\{\langle 0,1\rangle,\langle 1,1\rangle,\langle 1,0\rangle\}$, and this is the domain we use for weak Kleene based on the track down operations. $\{\langle 1,1\rangle,\langle 1,0\rangle\}$ is the tolerantly designated set, and $\{\langle 1,0\rangle\}$ is the strict truth value set, since $\langle 1,0\rangle$ is the only exact member of the upbiclosed set. We thus get the structure shown in Figure 3, where we repeat the Classical Logic structure from before, for convenience.

Both $A \wedge \neg A \Rightarrow B$ and $A \Rightarrow B \vee \neg B$ are validated in the classical structure in Subfigure 3a, so they must also be validated in the strict/tolerant structure of Subfigure $3 \mathrm{~b}$ using the weak Kleene truth tables. $A \wedge \neg A \Rightarrow B$ is validated because for every valuation $v, v^{t}(A \wedge \neg A)$ can never be $\langle 1,0\rangle$, the only strict truth value. $A \Rightarrow B \vee \neg B$ is validated because $v^{t}(B \vee \neg B)$ can never be $\langle 0,1\rangle$, and so must be tolerantly designated.

Example 8.2 (Four Valued Morgan Lattice, Version One) Figure 4 shows a four valued lattice in Subfigure 4a. Also shown is an up set (in fact, it is a prime filter), which will play a role later. In the Hasse diagram the ordering is upwards. The lattice structure is bounded, and distributive. Subfigure $4 \mathrm{~b}$ specifies an operation, Involution One. Thus we are given a Morgan algebra which, for convenience, we will call Four1

The Four1 structure is not orthocomplemented. For instance, $a \sqcup \bar{a}=a \sqcup a=a \neq 1$. It follows that in the corresponding bilattice product, Four $1 \odot$ Four1, the exact values are not closed under the generalized weak Kleene operations. Working through the details, the exact values of the product bilattice are $\langle 0,1\rangle,\langle 1,0\rangle,\langle a, a\rangle$, and $\langle b, b\rangle$. Now, $\|\langle a, a\rangle\|=\langle a, a\rangle \oplus \neg\langle a, a\rangle=\langle a, a\rangle$, and similarly $\|\langle b, b\rangle\|=\langle b, b\rangle$. Also $\langle a, a\rangle \wedge\langle b, b\rangle=\langle a \sqcap b, a \sqcup b\rangle=\langle 0,1\rangle$. Then, $\langle a, a\rangle \wedge^{c}\langle b, b\rangle=$ $(\langle a, a\rangle \wedge\langle b, b\rangle) \otimes\|\langle a, a\rangle\| \otimes\|\langle b, b\rangle\|=\langle 0,1\rangle \otimes\langle a, a\rangle \otimes\langle b, b\rangle=\langle 0,0\rangle$. Since $-\langle 0,0\rangle=\langle 1,1\rangle$, we have that $\langle 0,0\rangle$ is consistent, but not exact. We thus have a concrete example showing the exact values arising from Four1 are not closed under our weak Kleene generalization.

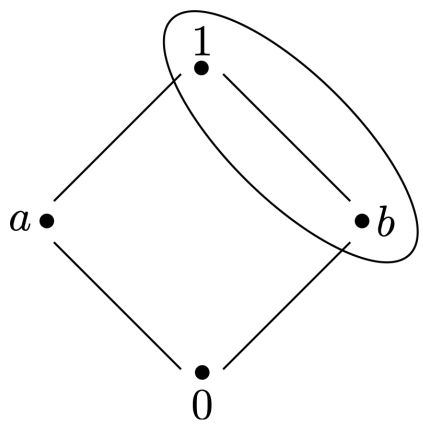

(a) A Four Valued Lattice

\begin{tabular}{c|c}
$x$ & $\bar{x}$ \\
\hline 0 & 1 \\
1 & 0 \\
$a$ & $a$ \\
$b$ & $b$
\end{tabular}

(b) Involution One

\begin{tabular}{c|c}
$x$ & $\bar{x}$ \\
\hline 0 & 1 \\
1 & 0 \\
$a$ & $b$ \\
$b$ & $a$
\end{tabular}

(c) Involution Two

Figure 4: Four Valued Morgan Lattices 


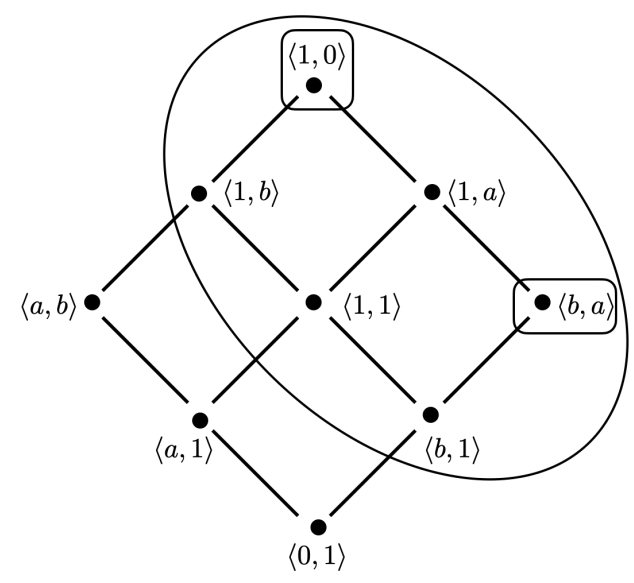

Figure 5: A Nine Element Strict/Tolerant Structure

Example 8.3 (Four Valued Morgan Lattice, Version Two) We continue drawing on Figure 4 , using the lattice from Subfigure 4a, but with Subfigure 4c specifying an alternative operation, Involution Two. This gives us a second Morgan algebra which we will call Four2. This time the Morgan algebra is easily seen to be orthocomplemented and so the construction from Section 7 applies.

The bilattice Four $\odot$ Four2 contains 16 members: $\langle 0, b\rangle,\langle 0, a\rangle,\langle 0,0\rangle,\langle a, 0\rangle,\langle b, 0\rangle$ are consistent but not exact; $\langle a, 1\rangle,\langle b, 1\rangle,\langle 1,1\rangle,\langle 1, b\rangle,\langle 1, a\rangle$ are anticonsistent but not exact; and $\langle a, b\rangle,\langle b, a\rangle,\langle 0,1\rangle,\langle 1,0\rangle$ are exact; $\langle a, a\rangle$ and $\langle b, b\rangle$ do not fall into any of these categories. Figure 5 shows the nine anticonsistent members, ordered upwards using the truth ordering from Four2 $\odot$ Four2. The large circle contains the tolerantly designated values, those members that are also in the upbiclosed $\{1, b\} \times\{1, b, 0, a\}$. The two members in squares, together, make up the strictly designated set, those members of the tolerantly designated set that are exact in Four2 $\odot$ Four2.

The many valued structure from Figure 4, using Involution Two as shown in Subfigure 4c, validates the same formulas as the strict/tolerant structure in Figure 5 using the track down operations. The track down operations, generalizing weak Kleene, are not much known. I point out that their behavior, and that of the cut down operations, is generally more complex than for the lattice based operations. Here is one example. In the structure of Figure 5 the lattice version of conjunction with $\langle 0,1\rangle$ has a simple behavior. For every $x$ in the structure, $x \wedge\langle 0,1\rangle=\langle 0,1\rangle$. But using the track down operation $\wedge^{t}$ we have the following.

$$
\begin{aligned}
\langle 0,1\rangle \wedge^{t}\langle 0,1\rangle=\langle a, b\rangle \wedge^{t}\langle 0,1\rangle=\langle b, a\rangle \wedge^{t}\langle 0,1\rangle & =\langle 1,0\rangle \wedge^{t}\langle 0,1\rangle=\langle 0,1\rangle \\
\langle a, 1\rangle \wedge^{t}\langle 0,1\rangle=\langle 1, a\rangle \wedge^{t}\langle 0,1\rangle & =\langle a, 1\rangle \\
\langle b, 1\rangle \wedge^{t}\langle 0,1\rangle= & \langle 1, b\rangle \wedge^{t}\langle 0,1\rangle=\langle b, 1\rangle \\
\langle 1,1\rangle \wedge^{t}\langle 0,1\rangle & =\langle 1,1\rangle
\end{aligned}
$$

Example 8.4 (Non-Distributivity) All the examples so far have involved distributive lattices. We conclude with an example that does not, shown in Figure 6 . The lattice is nondistributive because, for instance, $a \sqcap(b \sqcup c)=a \sqcap 1=a$ but $(a \sqcap b) \sqcup(a \sqcap c)=0 \sqcup 0=0$.

We form a strict/tolerant version for this, so that we may consider the behavior of our weak Kleene generalization. First we construct the bilattice product of the lattice with itself. 
This contains 36 members, and we do not provide a Hasse diagram for it. For the record, the anticonsistent part contains 15 members, namely $\langle a, b\rangle,\langle b, a\rangle,\langle c, d\rangle,\langle d, c\rangle,\langle 1,0\rangle,\langle 0,1\rangle,\langle a, 1\rangle$, $\langle 1, a\rangle,\langle b, 1\rangle,\langle 1, b\rangle,\langle c, 1\rangle,\langle 1, c\rangle,\langle d, 1\rangle\langle 1, d\rangle,\langle 1,1\rangle$ and this is our domain of strict/tolerant truth values, with the first 6 being exact.

As an upwardly closed subset of the lattice from Subfigure 6a we use $\{d, 1\}$, though different choices are certainly possible. This does happen to be a filter, but it is not prime. In fact, the lattice shown has no prime filters. The verification is simple. Any filter must contain 1, and so it must contains $a \sqcup b$ and $c \sqcup d$. If the filter were prime, it would have to contain one of $a, b$ and one of $c, d$. But then it would have to contain their meet, which is 0 , and so the filter would not be proper.

The up set $\{d, 1\}$ on the lattice induces an upbiclosed set on the product bilattice we constructed, the 12 element set $\{\langle 1,1\rangle,\langle 1, a\rangle,\langle 1, b\rangle,\langle 1, c\rangle,\langle 1, d\rangle,\langle 1,0\rangle,\langle d, 1\rangle,\langle d, a\rangle,\langle d, b\rangle,\langle d, c\rangle,\langle d, d\rangle$, $\langle d, 0\rangle\}$. The restriction of this to our anticonsistent domain gives us our tolerantly designated set, $\{\langle d, c\rangle,\langle 1,0\rangle,\langle 0,1\rangle,\langle 1, a\rangle,\langle 1, b\rangle,\langle 1, c\rangle,\langle d, 1\rangle\langle 1, d\rangle,\langle 1,1\rangle\}$. The further restriction of this to the exact part provides our strict set of truth values, $\{\langle d, c\rangle,\langle 1,0\rangle,\langle 0,1\rangle\}$.

Since distributivity fails in the many valued structure of Figure 6 it must fail in some way in the strict/tolerant structure just constructed. In fact, the sequent $X \wedge(Y \vee Z) \Rightarrow(X \wedge Y) \vee(X \wedge Z)$ is validated, using the generalized weak Kleene operators. But the sequent $(X \wedge Y) \vee(X \wedge Z) \Rightarrow$ $X \wedge(Y \vee Z)$ is not. There are several counterexamples; here is one. The valuation given by $v(X)=\langle b, a\rangle, v(Y)=\langle d, c\rangle, v(Z)=\langle c, d\rangle$ extends so that $v^{t}((X \wedge Y) \vee(X \wedge Z))=\langle 0,1\rangle$ and $v^{t}(X \wedge(Y \vee Z))=\langle b, a\rangle$, and $\langle 0,1\rangle$ is strictly designated but $\langle b, a\rangle$ is not tolerantly designated.

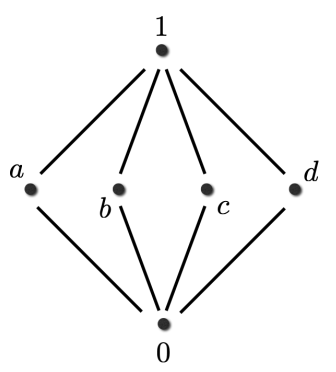

(a) A Non-Distributive Lattice

\begin{tabular}{c|c}
$x$ & $\bar{x}$ \\
\hline$a$ & $b$ \\
$b$ & $a$ \\
$c$ & $d$ \\
$d$ & $c$ \\
0 & 1 \\
1 & 0
\end{tabular}

(b) Its Involution

Figure 6: A Non-Distributive Morgan Algebra

\section{Conclusion}

After establishing the existence of a family of strict/tolerant logics based on weak Kleene generalizations, the next natural step should probably be to see if it can be made the basis of a version of the strict/tolerant heirarchy, as was the case with the generalizations examined in 12 . I have stopped short of that step. For one thing, almost certainly the work would proceed by exactly the same steps as before, and thus there would be nothing substantially new to be learned. The significant new thing here is really the determination of what additional structure needs to be added to the generalized weak Kleene family that was already proposed some years ago. Once that has been worked through, unless I'm missing something fundamental, the significant work is over.

An anonymous referee for this paper proposed an interesting direction for further research. The methods discussed here begin with logics characterized directly by lattices, and wind up 
with generalized versions of weak Kleene logic. Would it be possible to begin with a logic that was characterized by applying cut down or track down operations in a bilattice? To quote a specific case posed by the referee, "can the techniques implemented by the author be used to arrive at a strict-tolerant non-transitive counterpart to the paracomplete three-valued weak Kleene logic? A similar answer can be posed regarding the paraconsistent weak Kleene logic. And, of course, analogous questions can be raised regarding subsystems of these logics which also have ties to bilattices and cut or track down operations - like Deutsch's logic $S_{\text {fde }}$ and its dual, among others." This is a very good question, to which I have no answer. Perhaps others will have insights that can address it.

There still remain the philosophical and conceptual issues. The logic ST has generated much discussion on a range of foundational questions: what is a logic, what does it mean for logics to be the same or different, can ST substitute for classical logic in dealing with various paradoxes, and so on. The work in this paper does not attempt to address any of these important issues. It simply aims to show (again) that the ideas behind strict/tolerant logic are much more general than the original setting would suggest. The machinery of all this is interesting. Its significance is not yet so clear.

\section{References}

[1] Ofer Arieli and Arnon Avron. "The Value of Four Values". In: Artificial Intelligence 102 (1998), pp. 97-141.

[2] Eduardo Barrio, Federico Pailos, and Damian Szmuc. "A Hierarchy of Classical and Paraconsistent Logics". In: Journal of Philosophical Logic 49 (2020), pp. 93129. URL: https://doi.org/10.1007/s10992-019-09513-z.

[3] Eduardo Barrio, Lucas Rosenblatt, and Diego Tajer. "The logics of strict-tolerant logic". In: Journal of Philosophical Logic 44.5 (2015), pp. 551-571.

[4] D. A. Bochvar. "Ob odnom trechznacnom iscislenii i ego primenenii k analizu paradoksov klassiceskogo rassirennogo funkcional'nogo iscislenija". In: Matematiceskij Sbornik 4 (1938). English translation in [5], pp. 287-308.

[5] D. A. Bochvar and Merrie Bergmann. "On a three-valued logical calculus and its application to the analyses of the paradoxes of the classical extended functional calculus". In: History and Philosophy of Logic 2.87-112 (1981). English translation by Merrie Bergmann of [4].

[6] Pablo Cobreros et al. "Tolerant, classical, strict". In: Journal of Philosophical Logic 41.2 (2012), pp. 347-385.

[7] Thomas M. Ferguson. "Cut-Down Operations on Bilattices". In: 2015 IEEE International Symposium on Multiple-Valued Logic. 2015, pp. 24-29.

[8] Melvin C. Fitting. "Bilattices and the theory of truth". In: Journal of Philosophical Logic 18 (1989), pp. 225-256.

[9] Melvin C. Fitting. "Kleene's three-valued logics and their children". In: Fundamenta Informaticae 20 (1994), pp. 113-131.

[10] Melvin C. Fitting. "Bilattices are nice things". In: Self-Reference. Ed. by Thomas Bolander, Vincent Hendricks, and Stig Andur Pederrsen. Center for the Study of Language and Information, 2006. Chap. 3, pp. 53-77. 
[11] Melvin C. Fitting. "Bilattice Basics". In: Journal of Applied Logics - IfCoLog Journal 7.6 (2020). (Will also appear in [14].), pp. 973-1018.

[12] Melvin C. Fitting. "A Family of Strict/Tolerant Logics". In: Journal of Philosophical Logic 50 (2021). First published online, September 7, 2020., pp. 363-394. URL: https://doi.org/10.1007/s10992-020-09568-3.

[13] Melvin C. Fitting. "Modal, Fuzzy, ..., Vanilla, Fixpoint Theories of Truth: A Uniform Approach". To appear in a forthcoming volume, Kripke On Modal Logic. 2021.

[14] Melvin C. Fitting. "Bilattice Basics". In: Philosophical Logic. Ed. by Ali Sadegh Daghighi et al. Landscapes in Logic. (Has already appeared as [11].) College Publications, 2021 forthcoming.

[15] Melvin C. Fitting. "The Strict/Tolerant Idea and Bilattices". In: Arnon Avron on Semantics and Proof Theory of Non-Classical Logics. Ed. by Ofer Arieli and Anna Zamansky. Outstanding Contributions to Logic. Springer Nature, 2021 (forthcoming).

[16] Stephen C. Kleene. Introduction to Metamathematics. Princeton, NJ: D. Van Nostrand, 1950.

[17] Saul Kripke. "Outline of a theory of truth". In: The Journal of Philosophy 72.19 (1975). Reprinted in New Essays on Truth and the Liar Paradox, R. L. Martin, ed., Oxford (1983), pp. 690-716.

[18] Francesco Paoli amd Michele Pra Baldi. "Proof theory of paraconsistent weak Kleene logic". In: Studia Logica 108 (2020), pp. 779-802.

[19] David Ripley. "Paradoxes and Failures of Cut". In: Australasian Journal of Philosophy 91.1 (2013), pp. 139-164.

[20] Chris Scambler. "Classical logic and the strict tolerant hierarchy". In: Journal of Philosophical Logic 49 (2020), pp. 351-370. URL: https://doi.org/10.1007/ s10992-019-09520-0.

[21] Yang Song, Hitoshi Omori, and Satoshi Tojo. "A two-valued semantics for infectious logics". In: Proceedings of ISMVL. 2021 (forthcoming).

[22] Damian E. Szmuc. "Track-down operations on bilattices". In: Proceedings of the 48th IEEE International Symposium on Multiple-Valued Logic. Ed. by Robert Wille and Martin Lukac. 2018, pp. 74-79.

[23] Damian E. Szmuc. "An Epistemic Interpretation of Paraconsistent Weak Kleene Logic". In: Logic and Logical Philosophy 28 (2019), pp. 277-330.

[24] Damian E. Szmuc and Thomas M. Ferguson. "Meaningless Divisions". In: Notre Dame Journal of Formal Logic Forthcoming (2021).

[25] Elia Zardini. "Naive modus ponens". In: Journal of Philosophical Logic 42.4 (2013), pp. $575-593$. 